\title{
OpenSky Report 2017: Mode S and ADS-B Usage of Military and other State Aircraft
}

\author{
Matthias Schäfer*, Martin Strohmeier ${ }^{\dagger}$, Matthew Smith ${ }^{\dagger}$, Markus Fuchs ${ }^{\ddagger}$, \\ Vincent Lenders ${ }^{\S}$, Marc Liechti ${ }^{\uparrow}$, Ivan Martinovic ${ }^{\dagger}$ \\ *University of Kaiserslautern, Germany \\ ${ }^{\dagger}$ University of Oxford, UK $\quad{ }^{\ddagger}$ SeRo Systems, Germany \\ schaefer@cs.uni-kl.de \\ firstname.lastname@cs.ox.ac.uk_fuchs@sero-systems.de \\ $\S$ armasuisse, Switzerland \\ IOpenSky Network, Switzerland \\ vincent.lenders@armasuisse.ch liechti@opensky-network.org
}

\begin{abstract}
This paper provides up-to-date statistics on SSR Mode S and 1090ES ADS-B usage by military and state aircraft from the OpenSky Network. By analysing the large host of real-world data collected by OpenSky using more than $\mathbf{7 0 0}$ receivers, we provide insights on the equipage of these state aircraft, their capabilities, usage patterns and much more. We publish up-to-date, empirically validated numbers on the status of the ongoing ADS-B deployment based on data collected over large areas in Europe, North America, and other continents, and categorize these aircraft by incorporating publicly available data sources. Our measurements indicate that ADS-B equipage of military aircraft is $\mathbf{4 2 . 9 \%}$, which is considerably less than ADS-B deployment in civil aviation. While there is considerable variation between countries, our results show that there is a long way to go to for a safe and efficient integration of military and state air traffic into the next generation civil air transportation system.
\end{abstract}

\section{INTRODUCTION}

Historically, governmental and military aircraft (collectively called state aircraft) have been strictly distinguished from civil air traffic. In Article 3 of the Convention on International Civil Aviation [1], the International Civil Aviation Organization (ICAO) has expressly excluded state aircraft from its regulations. However, facing today's fast increase in air traffic, aviation authorities and organizations have recognized that a harmonized approach to managing civil and state aircraft is inevitable to meet both the needs of international air traffic flows and national security [2]. Especially in congested airspaces, harmonized regulations including communications, navigation, and surveillance are a precondition for safely and efficiently accommodating state aircraft into civil airspace systems.

In many airspaces around the world, the Secondary Surveillance Radar (SSR) Mode S has become the primary data link for civil air traffic surveillance and collision avoidance. Moreover, the SSR Mode S downlink has been selected as the globally interoperable data link of the next generation civil air traffic surveillance technology Automatic Dependent Surveillance-Broadcast (ADS-B) [3]. As ADS-B becomes mandatory in many parts in the world in the late 2010s (Aus- tralie 1 ) and early 2020s (US ${ }^{2}$ and Europe ${ }^{3}$, many airlines have already started updating their fleet with ADS-B capabilities. About $70 \%$ of all airliner Mode S transponders used in the US and Europe reported having ADS-B capabilities in 2016 [4]. As a consequence, equipping state aircraft with SSR Mode $\mathrm{S}$ and ADS-B transponders is absolutely essential to enable interoperability with civil air traffic. The European Union has in fact mandated the equipage with SSR Mode S transponders for all aircraft operating in accordance with instrument flight rules-including state aircraft ${ }^{4}$

While there is an obvious consensus to equip state aircraft with civil surveillance technologies, the extent to which they support and use SSR Mode S and ADS-B remains unclear. Even though the support of civil technologies has not only a direct impact on operations, but also important privacy implications for the stakeholders, there is no public study on real-world usage of SSR Mode S and ADS-B by state aircraft available.

This paper fills this gap by providing an overview of the current use of SSR Mode S and ADS-B by state aircraft around the world. More specifically, we analyse a vast record of SSR Mode S downlink and ADS-B communications collected by hundreds of receivers of the OpenSky Network around the world. By combining OpenSky's data with an extensive aircraft operator data set that we collected from different data sources in the Internet, we provide a quantitative overview of SSR Mode S and ADS-B state aircraft equipage and usage in different parts of the world. With this study, we build the foundations for an up-to-date assessment of the ongoing international harmonization of surveillance technologies, the interoperability of state aircraft with civil air traffic, and the readiness of state aircraft for the Next Generation Air Transportation System.

The harmonization, however, comes at a price. By using civil technologies, state aircraft might reveal information not meant for the public such as the leakage of information on

\footnotetext{
${ }^{1}$ Instrument number CASA 61/14

${ }^{2}$ Code of Federal Regulations $\S 91.225$

${ }^{3}$ Commission Implementing Regulation (EU) No 1028/2014

${ }^{4}$ Commission Implementing Regulation (EU) No 1207/2011
} 


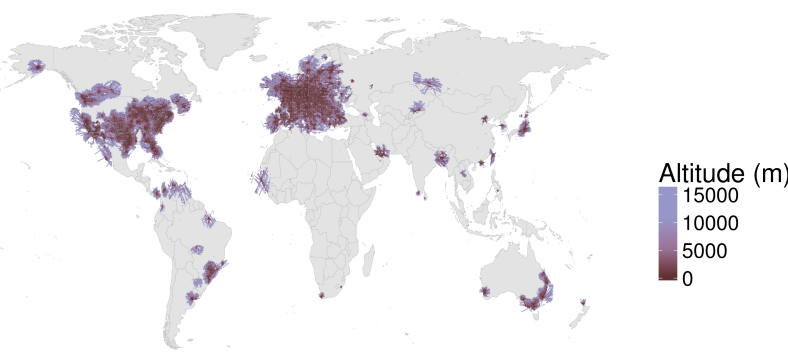

Figure 1: OpenSky's coverage on the 7th of June, 2017. The color indicates the lowest altitude observed in that region. It is an indicator for the receiver density, since the reception at low altitudes requires a nearby receiver due to the limited line of sight.

drone operations through commercial tracking services ${ }^{5} \mathrm{We}$ therefore discuss and highlight the potential privacy implications as a result of this harmonization.

We first discuss our data: the OpenSky Network in Section [II aviation meta data sources in Section [III, and a description of our concrete data sets in Section IV] Using this data, we provide an analysis of Mode S and ADS-B usage by military and state aircraft in Section V. Section VI provides a concrete case study of real-world military transponder use while Section VII discusses the privacy effects of such usage and analyses current mitigation tactics. In Section $[\mathrm{VIII}$ we discuss future plans of the OpenSky Network before concluding in Section IX

\section{THE OPENSKy NeTWORK}

The OpenSky Network ${ }^{6}$ is a crowd-sourced sensor network collecting air traffic control (ATC) data. Its objective is to make real-world ATC data accessible to the public and to support the development and improvement of ATC technologies and processes. Since 2012, it continuously collects air traffic surveillance data. Unlike commercial flight tracking networks (e.g., Flightradar24, FlightAware, Plane Finder), the OpenSky Network keeps the raw Mode S messages as they are received by the sensors in a large historical data store and the data is available to researchers and analysts from different areas for free. For more information on OpenSky's history, architecture and use cases refer to [4], [5], [6].

The network started out with eight sensors in Switzerland and Germany and has grown to more than 700 currently active receivers at locations all around the world. As Figure 1 shows, the network's extensive coverage has expanded over large areas in Europe and North America, but also includes a large number of sites in Africa, Asia, Oceania, and South America.

\footnotetext{
${ }^{5}$ e.g. https://goo.gl/jzj9JF

$6 \mathrm{http}: / /$ opensky-network.org
}

As of this writing, OpenSky's data set contains four years of ATC communication data. Besides the payload of each Mode $\mathrm{S}$ downlink message, OpenSky stores additional metadata. Depending on the receiver hardware, this includes precise timestamps for the signal arrivals, receiver location, and signal strength. While the network initially focused on ADS-B only, it extended its data range to the full Mode S downlink channel in March 2016. Eight months later, in November 2016, the network additionally opened its servers to anonymous feeders without the need for setting up an account. Since then, the data set has been growing exponentially. Currently, it contains more than 2 trillion Mode S or ADS-B transmissions from more than 200,000 transponders and it grows by about 15 billion transmissions every day. The growth of the data set and the timeline is depicted in Figure 2

Although there is a certain risk for noise in OpenSky's data, e.g., due to ADS-B spoofing (see [6] for details) or receivers sharing fake data, the high density of receivers and overlapping coverages allow for cross-checking the data over multiple sites [7]. In this way, non-existent aircraft and receivers sharing fake data are identified and filtered. However, while a certain level of noise is inevitable, we argue that the sheer amount of valid data keeps the noise ratio negligibly low. For example, launching ADS-B spoofing attacks at a scale large enough to bypass the filters and effect our results is rather unlikely since the exact receiver sites are unknown to outsiders.

\section{Aviation Meta Data Sources}

In order to provide context to aircraft movements we use aviation meta data such as aircraft operator, type, and category. This section discusses the availability and collection methods of the sources required for our large-scale analysis in the remainder of this work.

A number of public data sources exist which provide aircraft meta-information based on different identifiers. These identifiers include aircraft registration or the unique 24-bit ICAO Mode S transponder address. The data usually includes type and the owner/operator, which can then be used for further in-depth analysis and stakeholder identification. We use several of these third-party databases in our analysis of aircraft meta data of Mode S and ADS-B users:

- The Planeplotter software [8], with a version from October 2016. This contains 147,084 rows of aircraft data.

- Junzi Sun at TU Delft has collected all visible aircraft from a public flight tracker for some time. At the time of writing, we used a data set collected over a period of 18 months and amounting to 116,338 rows [9].

- Airframes.org provides comprehensive aircraft and owner data for many aircraft; Usually, this also includes background knowledge (e.g. pictures and historical ownership information [10]). This is particularly useful 


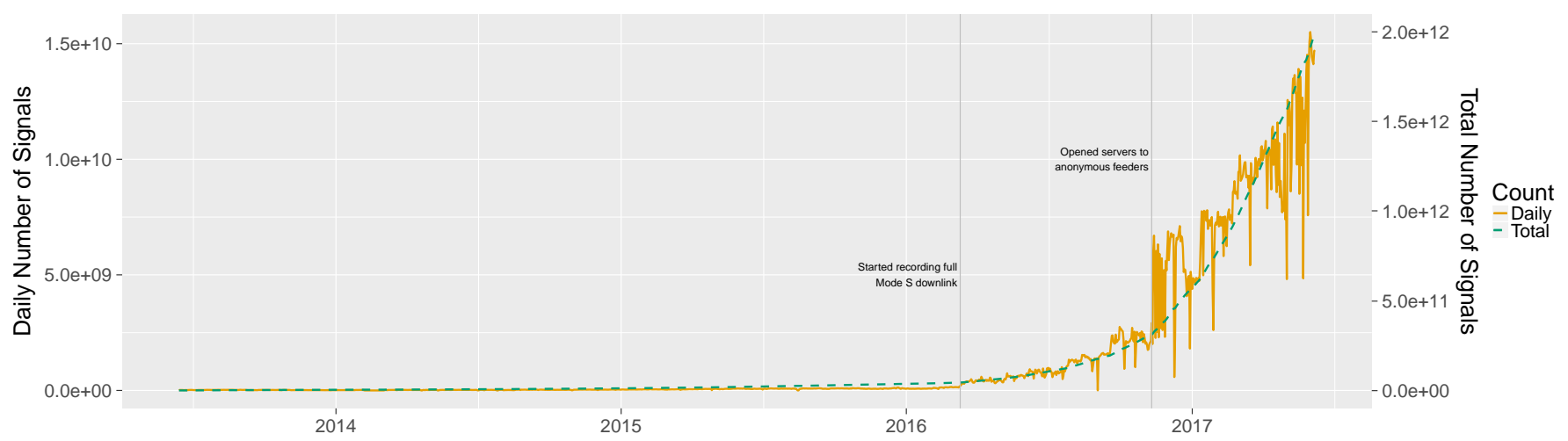

Figure 2: The growth of OpenSky's data set over time from June 2013 to June 2017.

\begin{tabular}{|l|c|c|}
\hline Aircraft Affiliation & Aircraft & {$[\%]$} \\
\hline Business & 97,372 & $9.3 \%$ \\
\hline Military & 519,235 & $49.6 \%$ \\
\hline State-related & 2,680 & $0.3 \%$ \\
\hline Scheduled/General/Other & 424,746 & $40.6 \%$ \\
\hline Ground/Airport & 1,785 & $0.2 \%$ \\
\hline
\end{tabular}

Table I: Description of our data set containing metadata of 1,045,818 aircraft ICAO IDs. It is worth noting that, as a result of adding whole blocks of IDs to the list, not all these IDs belong to existing aircraft. This data set is later joined with OpenSky's data which reduces it to a set of 6024 military aircraft that were actually operated during the considered period.

in settling uncertainty on aircraft meta data.

- Aircraft registered in the USA are logged on a dailyupdated FAA database, containing owner records. This is online and available for download but exclude any sensitive owner information. Even so, the data set used for this work contained 320,777 records, primarily used in March 2017 [11].

- The plane spotting and aviation community actively maintains databases with spotted aircraft. They usually operate Mode S receivers (typically SBS-1 or dump1090) and enrich the received data with information such as operator, type, or registration manually. The BaseStation.sqb database structure of Kinetic Avionic's SBS-1 BaseStation software has become the de facto standard format and is also used to exchange and share their databases in forums and discussion boards.

Table 1 breaks down the aircraft types collected in our data set by their affiliation. The type is determined by the registrar and the type of the aircraft, i.e., if a non-airline business is the registrar then it is classed as a business aircraft.

Since these sources are comprised often by hand or from other smaller sources, they are noisy in terms of deprecated or false information. This is due to aircraft changing registration

or undergoing transfer over time. However, generally they tend to provide a sufficiently accurate picture of the aviation environment. The noise is further reduced by cross-checking the data between multiple sources and settling uncertainties manually.

\section{A. Military Aircraft Identification}

Identification of military aircraft is not limited to those contained in the obtained public data sources. Air forces typically reserve a block in their country's ICAO ID range for military transponders; for example, identifiers used by the US Air Force tend to begin with 'AE'. Any aircraft with an ICAO ID matching this pattern can be identified as used for military purposes. Exploiting this information, we can identify aircraft not in our public meta data sources-including the country and operator-though in these instances we lack additional meta information such as aircraft type. As Table I shows, this approach resulted in a list of about 520 thousand military aircraft.

\section{B. State Aircraft Identification}

In addition to-and partly overlapping with—aircraft operated by the military, we analyse aircraft operated by, or for, a state. Examples of such aircraft used by the executive branch include government aircraft, police aircraft, border control aircraft, or the coast guard.

We constructed a list of relevant aircraft of interest to conduct our analysis. To do this, we obtained the unique 24bit ICAO identifier of government-related aircraft using the sources described above and searching for keywords such as 'government'. We then verified the accuracy of all potential aircraft by hand using online sources, from official data over social networks to lists maintained by aviation enthusiasts. In total, this resulted in a list of 2,680 state aircraft (see Table 【).

\section{The Data Set}

In order to analyse Mode S and ADS-B usage of state aircraft, we considered the set of all $1090 \mathrm{MHz}$ downlink transponder transmissions recorded by OpenSky in April 2017. The set comprises about 290 billion transmissions which 


\begin{tabular}{|l|c|c|}
\hline Aircraft Type & Aircraft & {$[\%]$} \\
\hline Fighter / Attack Aircraft & 409 & $6.8 \%$ \\
\hline Trainer Aircraft & 1356 & $22.5 \%$ \\
\hline Tanker Aircraft & 325 & $5.4 \%$ \\
\hline Transport Aircraft & 1691 & $28.1 \%$ \\
\hline Business Jets & 486 & $8.1 \%$ \\
\hline Surveillance/Reconnaissance Aircraft & 214 & $3.6 \%$ \\
\hline UAV & 12 & $0.2 \%$ \\
\hline Helicopters & 924 & $15.3 \%$ \\
\hline Other/Uncategorized & 607 & $10.1 \%$ \\
\hline Sum of all Aircraft & 6024 & $100 \%$ \\
\hline
\end{tabular}

Table II: Breakdown of military aircraft seen by OpenSky in April 2017.

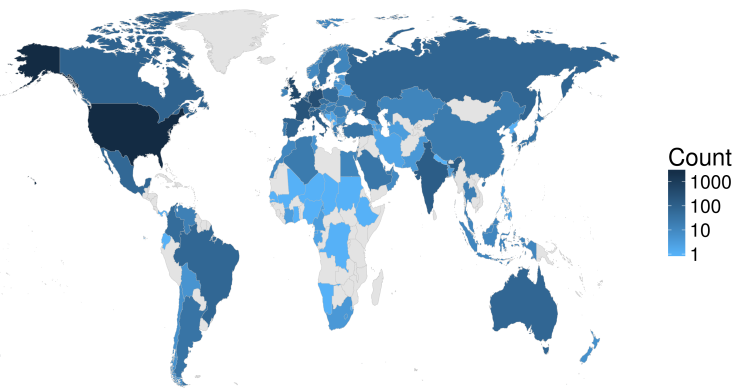

Figure 3: Countries of origin of the transponder-equipped military aircraft seen by OpenSky. Deeper colours represent a higher proportion of the total military aircraft seen.

altogether take up about 30 terabytes of disk space in OpenSky's back-end. After pre-processing the data to reduce noise induced by the receiving hardware and the radio environment, 125,000 different transponder IDs were left in the data set.

We assume that over the course of one month, enough military and state air traffic crossed OpenSky's coverage to allow us to produce representative statistics on their Mode $S$ and ADS-B usage. In fact, after combining OpenSky's data with the metadata, we found 6024 different military aircraft in the data set.

In a next step, we broke these aircraft into 9 different categories which are shown in Table II This allows us to consider these categories separately in our analysis. Note that these numbers only include aircraft which used Mode S/ADS$\mathrm{B}$ communication in our reception range and could be identified as belonging to a military institution via ICAO range or meta data sources as described in Section III-A. Furthermore, many aircraft types fulfil several different functions and could conceivably be part of more than one category (e.g., UAV being used for surveillance purposes); thus, we have assigned these cases to their category according to their typical main purpose. For 607 (10.1\%) of all military aircraft, we could not assign an aircraft type or categorize it into a listed group.

Figure 3 shows the countries of origin of the detected military and state aircraft as judged from their ICAO address range. United States comprised 3,410 of the aircraft (53.1\% of all state aircraft), followed by the UK (7.5\%), Germany (5\%) France (3.5\%), India (2\%), Italy (1.8\%), Belgium (1.6\%), the Netherlands $(1.4 \%)$, and the residual $24 \%$ by 90 other countries. Note, that this result is biased by OpenSky's focus on coverage in Europe and the US (see Figure 1).

As for other state aircraft, our approach returned a list of 401 verified government aircraft from 79 different states that were seen by OpenSky in April 2017. Similarly, we identified a further 214 aircraft that belonged to one of several staterelated surveillance categories: police (local or federal), coast and border guard, and other similar entities (see Table IV).

\section{Unmanned Aerial Vehicles}

Besides manned aircraft, we have also started to encounter UAVs within OpenSky. Naturally, as UAVs are poised to enter the civil airspace, some utilize transponders to be able to move safely and cooperate with air traffic control. Consequently, they are visible to flight trackers and ADS-B/Mode S sensors in general. We analysed the historical data of OpenSky based on our publicly available aircraft information to see whether we could find evidence of Mode S and ADS-B-equipped UAVs.

In last year's report [4], the only discovered evidence was of ADS 95 Ranger Drones used by the Swiss Airforce for surveillance and border patrol. We have as of this year encountered 12 of these UAVs, which, whether deliberate or not, use Mode $\mathrm{S}$ to communicate their identification and altitude and can subsequently be localised using multilateration by interested flight trackers.

Additionally, we have now received signals from two General Atomics MQ-9 Reaper UAV as well as three Northrop Grumman RQ-4 Global Hawk. Some of these sightings have also been reported in dedicated aviation blogs, illustrating the fact that air traffic communication signal collection is widespread and thorough [12].

\section{Mode S \& ADS-B Usage Analysis}

With the ability to identify military and other state aircraft we can further analyse how they use Mode $\mathrm{S}$ and associated technologies. Specifically, we are interested in ADS-B and the Aircraft Collision Avoidance System (ACAS) as these are both safety-critical technologies. In this section we look at the extent to which Mode $\mathrm{S}$ is used by both groups as well as the manner in which they use it.

\section{A. Capabilities}

Initially, Mode $\mathrm{S}$ was developed as an efficient and reliable secondary surveillance radar technology providing accurate location and identity information to air navigation service providers. In classic Mode $S$, the two capability levels are defined: elementary surveillance (ELS) and enhanced surveillance (EHS) [13]. ELS compliant transponders must be able to provide altitude, flight status (airborne/on the ground), capability, and identity information. Transponders compliant with EHS must additionally support the interrogation of 8 


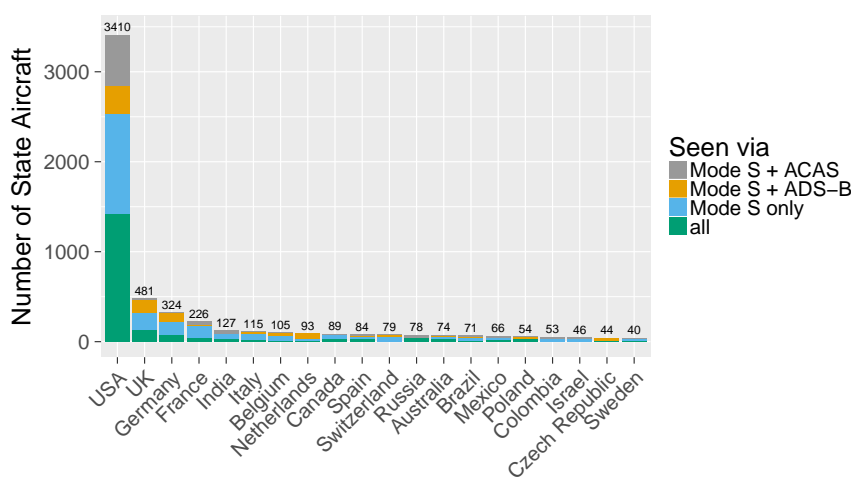

(a) Absolute

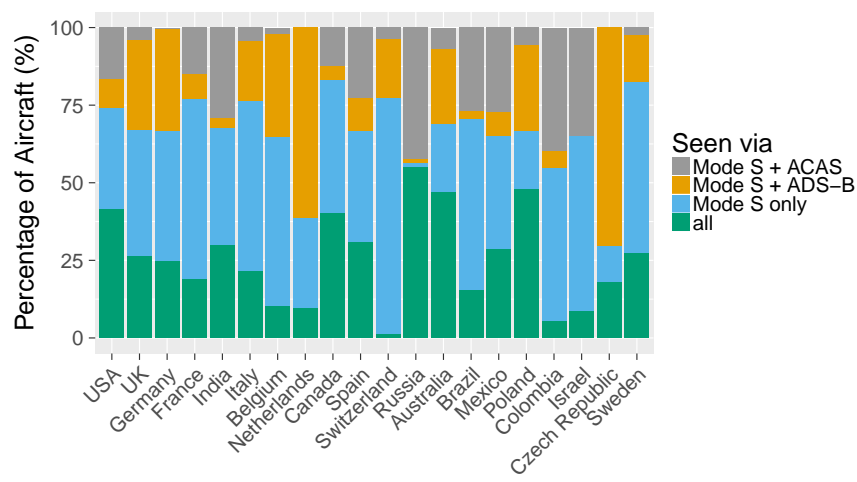

(b) Expressed as a percentage

Figure 4: Mode $S$ and associated technology use for state aircraft, by country.

so called Downlink Aircraft Parameters. These parameters include selected altitude, roll and track angle, heading, and speed (ground and airspeed). Whether a transponder is ELS or EHS compliant is indicated in the capability field of Mode $\mathrm{S}$ all-call replies (transponder level; refer to [4] for more details).

Besides elementary and enhanced surveillance, Mode S has also become the data link for other safety-critical applications such as airborne collision avoidance and the next generation air traffic surveillance technology ADS-B. Whether the aircraft is equipped with ACAS and ADS-B can be inferred simply by the downlink formats (DF) transmitted by the transponder. For instance, if a transponder, identified by its ICAO 24-bit transponder address, transmits DF 17 replies with encapsulated ADS-B messages, we can assume that the transponder has ADS-B capabilities. The same method can be used to detect ACAS capabilities (DF 0 and 16).

Since Mode $\mathrm{S}$ is the primary surveillance technology in many airspaces around the world, the transponder capabilities determine the extent to which state aircraft can be incorporated in civil air traffic. We therefore start our analysis with a closer look at the capabilities of state aircraft. We are particularly interested in the equipage status and whether there are differences between countries and aircraft categories as listed in Table I
1) Countries: Focussing on country of registration, Figure 4 displays absolute and percentage values of the observed Mode S transponder capabilities. For readability and statistical reasons, we limited this analysis to the top 20 countries with respect to the number of state aircraft in the data set. Here we see that the vast majority of aircraft observed originate from the US (3732), with the UK, Germany and France being the only other countries to have more than 250 .

Looking to the percentages (Figure 4b), we see that for most countries, less than $50 \%$ have been seen using Mode $\mathrm{S}$ for all three applications. In fact, only Russia has shown to use ADS-B and ACAS with 55.7\%. In contrast, only $1.2 \%$ of Switzerland's state aircraft have shown to be compliant with all civil surveillance technologies.

Other notable outliers are those with very low percentages $(<10 \%)$ of observed ACAS usage (Belgium, the Netherlands, and Switzerland), as well as Russia which has an extremely high percentage of ACAS users (97.5\%). As for ADS-B, the Czech Republic has the highest percentage $(83.3 \%)$ of state aircraft with observed ADS-B capabilities. The next in the line are Poland (76.8\%), Australia (74\%) and the Netherlands $(72 \%)$. All other countries have a much smaller percentages $(<50 \%)$ of state aircraft using ADS-B. The tail of ADS-B usage is made of Switzerland (21\%), Brazil and Japan (both around $18 \%$ ) and Colombia $(11.1 \%)$. The average over all countries is $42.9 \%$, which is considerably less than the civil average of around 70\% in Europe and the USA [4]. Whether this is by choice or a lack of deployment, it indicates that there is a long way to go to catch up with the ongoing ADS-B deployment.

2) Aircraft Categories: Figure 5 shows the equipment usage by category, both absolute and percentage values. Tanker aircraft have the biggest proportion of aircraft using all technologies at over $86.8 \%$ of those seen. Surveillance, transport and business aircraft have close to $50 \%$ usage of all technologies. Interestingly, UAVs stand out as they do not seem to use ADS-B at all. Another interesting observation is that helicopters and fighters do not seem to be equipped with ACAS. This, however, can most likely be attributed to the fact that they naturally rely on their visual awareness for collision avoidance and are therefore primarily equipped with technologies for ground-based surveillance.

3) $E H S$ vs. $A D S-B$ : As described above, some state/military aircraft are not bound by the same regulation with respect to ADS-B equipage. This is particularly interesting in the case of state aircraft which operate similarly to commercial aircraft and thus have an arguable need for ADS-B equipage. For existing aircraft, this requires some retrofit process, the most common of which is the installation of Mode S EHS capable transponders, which in turn allows but does not require ADS-B usage. Figure 6 shows that generally, updating transponders with ADS-B comes after EHS, but the support of EHS does not necessarily imply ADS-B capabilities. 


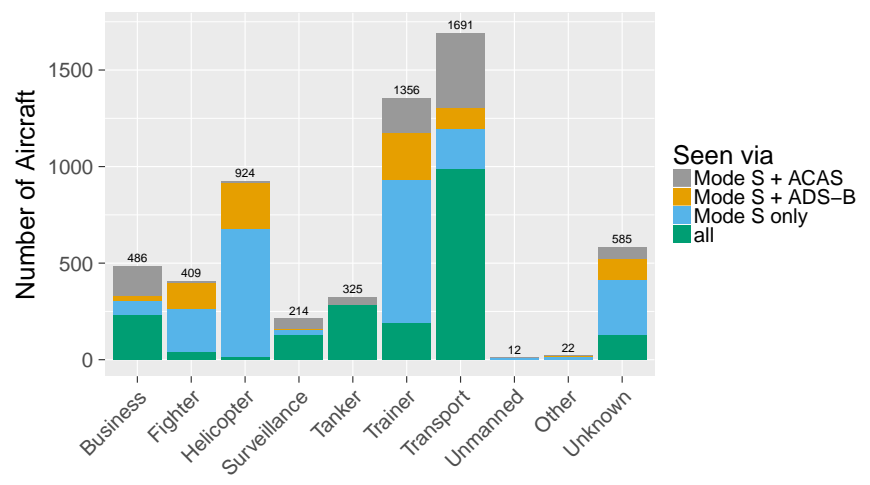

(a) Absolute values.

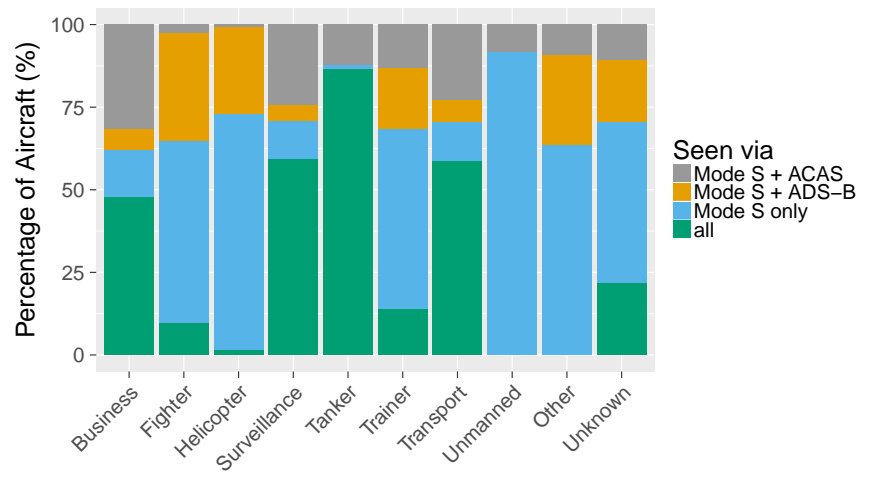

(b) Expressed as a percentage.

Figure 5: Mode $S$ and associated technology use for state aircraft, by category.

\section{B. ADS-B Usage}

As the deployment of the next generation air traffic surveillance system is progressing towards the full equipage of civil aircraft with ADS-B in many parts in the world, we continue our analysis with a closer look at those $42.9 \%$ of military and state aircraft which are equipped with ADS-B. In particular, we are interested in whether military and state aircraft are aware of nearby civil air traffic and to which extent they reveal information over ADS-B.

1) Uplink Capabilities: In order to infer whether nearby civil air traffic is included in the situational awareness of pilots, we take a closer look at the reported ADS-B uplink capabilities. ADS-B In provides an improvement in operational context by directly observing ADS-B Out messages in range; this can provide pilots with a better picture of nearby traffic without relying on ATC. This can be served either over the 1090ES or UAT links. In Figure 7 we compare 1090ES and UAT ADS-B In deployment. Clearly, a higher percentage of ADS-B In deployment for the countries observed lies with 1090ES - to be expected since 1090ES is the standard transponder type used for IFR flights. It is interesting to note that North American countries (USA and Canada) have a much bigger share of UAT usage, while many other countries seem to ignore UAT totally. This is consistent with the fact that UAT is mostly used by the North American general aviation than by other countries [4], [14]. Argentina is an extreme outlier since 26 of its 33 state aircraft reported having UAT ADS-B In functionality.

Interestingly, state aircraft seem to have a much higher percentage of aircraft with ADS-B In capabilities than civil aircraft. According to our last year's report [4], only 9.5 percent of the overall air traffic in OpenSky's coverage reported having 1090ES reception capabilities and 10.8\% UAT. In the military domain, $80.4 \%$ of the ADS-B equipped aircraft reported having 1090ES ADS-B In functionality and $14.6 \%$ UAT. This indicates a much higher tendency towards autonomous situational awareness in military aviation while civil aviation rather relies on ground-based ATC.

2) Provided information: Whilst ADS-B position broadcast is the most interesting, further data can also be transferred as part of the ADS-B protocol including callsigns and velocity. However, not all state aircraft transmit all data types. In fact, some choose not to transmit certain types at all. In Figure $8 \mathrm{a}$ we can see the percentage of time where different categories of aircraft transmit position and identity. In this we see three clear groups, irrespective of category: most identity, mostly position and both.

Those mostly transmitting identity with little position are in the top left and are the most dispersed group, i.e. many transmit position less than half the time. In contrast, those just broadcasting position but not identity typically do not broadcast identity at all. We also see a third group, namely those regularly transmitting both identity and position.

In the case of velocity against position (Figure 8b), we instead see two primary groups-those regularly transmitting both velocity and position, and those rarely transmitting either. For the former group, the vast majority transmit both at most opportunities. However, for the latter group, note that barely any aircraft regularly transmit velocity but not position. This could be due to position being a key ADS-B component, so the impetus for broadcast causing either constant or rare transmission. In general, we can conclude that velocity information is only provided along with position, but not necessarily the other way around. Only a negligible fraction of aircraft transmitted velocity information but did not reveal their position.

\section{CASe Study}

Figure 9 illustrates how ADS-B was used during a military transport flight. It was turned on at departure when the aircraft passed the $30,000 \mathrm{ft}$ altitude mark and turned off right before leaving the Greek airspace. It was later turned on again after the aircraft entered the Hungarian airspace and turned off right before the aircraft's final descent in Germany. After a layover of a little more than 2 hours, the aircraft departed westbound and started - as before - emitting ADS-B position only after it ascended above 30,000 ft. Interestingly, it stopped emitting ADS-B positions right before entering the UK airspace but then turned on its position broadcast function again a little 


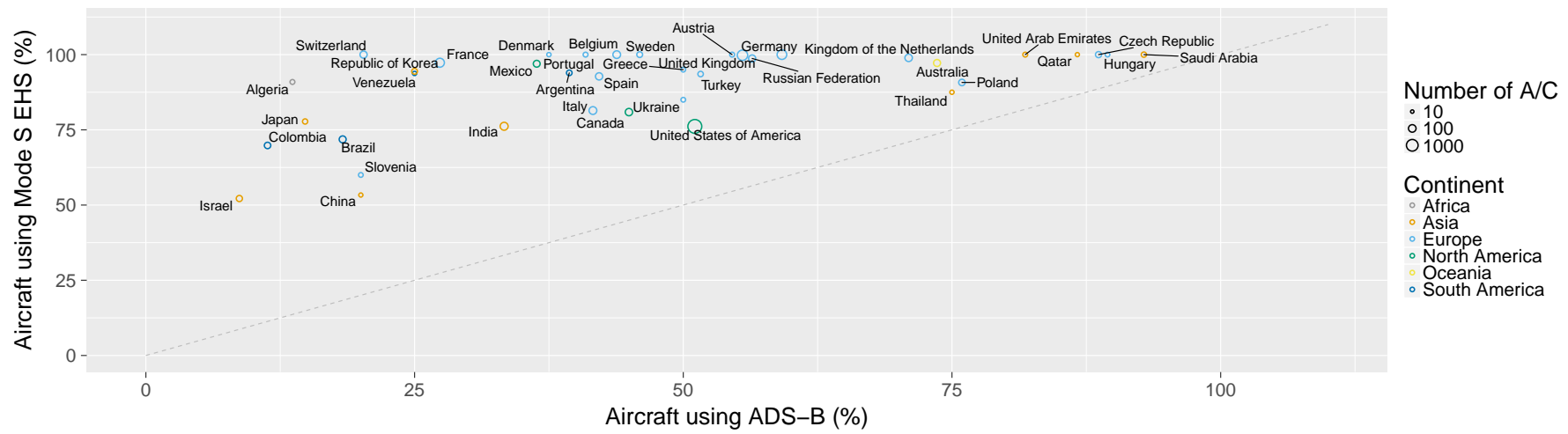

Figure 6: Percentage of state aircraft that used ADS-B versus percentage of state aircraft that reported having Mode S Enhanced Surveillance (EHS) capabilities, by country and continent. It is clear to see that the equipage with ADS-B comes after Mode S EHS.

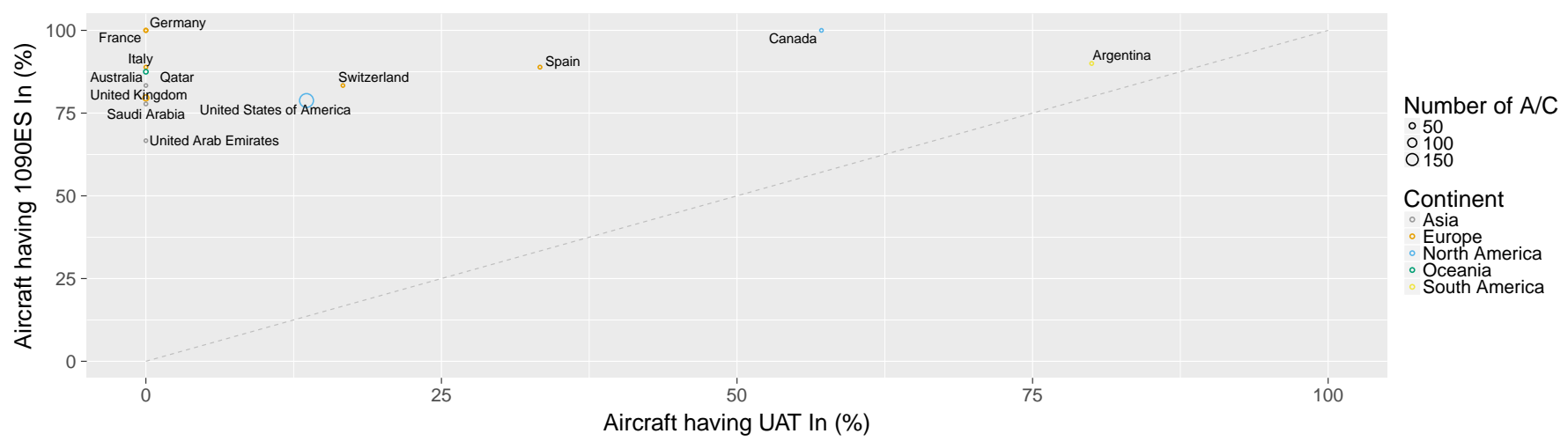

Figure 7: Percentage of state aircraft that reported having 1090ES ADS-B In capabilities versus percentage of state aircraft that reported having UAT ADS-B In capabilities, by country and continent. Apparently 1090ES ADS-B In has priority over UAT ADS-B In, which is natural since 1090ES ADS-B is the de facto standard for IFR flights.

later-still in the UK airspace. It finally stopped broadcasting its position when it left the UK mainland.

This behavior shows that ADS-B can and is turned on and off dynamically by military aircraft. On the one hand, turning it on at least sometimes indicates a general willingness to use ADS-B and, by doing so, facilitate tracking with civil surveillance technologies. On the other hand, turning it on only after reaching cruising altitude and turning it off again before descending most likely aims at concealing the airport of departure and arrival. In addition, for reasons unknown to us, they also turn off ADS-B when overflying certain airspaces.

\section{DISCUSSION}

As software-defined radios have proliferated in recent years and made it easy and cost-effective for any actor to receive air traffic communication, privacy in aviation has become an increasingly popular topic. A number of papers have addressed this in the ADS-B [15] and ACARS [16] scenarios, with respect to location privacy and tracking prevention.

\section{A. Display of State Aircraft on Commercial Trackers}

Besides these choices concerning the direct use of ADS-B transponders, air forces and also state governments belong to the interest groups which seek to restrict commercial internet databases displaying their aircraft's whereabouts publicly. One way in which they can do this is through the Federal Aviation Administration's (FAA) Aircraft Situation Display to Industry (ASDI) scheme [17]. The ASDI data feed provides a nearlive data source for aircraft currently seen by the FAA and is used by websites such as FlightRadar24 as one of many data sources. Non-commercial aircraft owners can request to have data shared about their aircraft via ASDI either restricted or completely blocked. Even though flight tracking sites can obtain data about these aircraft in other ways, websites such as Flightradar24 have taken this further, complying with these block lists even for data obtained from other sources [18].

Because of the requirement to register for a block, we claim that aircraft owners requesting one have a clear interest in privacy. However, crowd-sourced collection of ADS-B and Mode $S$ data demonstrates the ease of setting up a personal sensor. As such, we claim that these block lists are of limited 


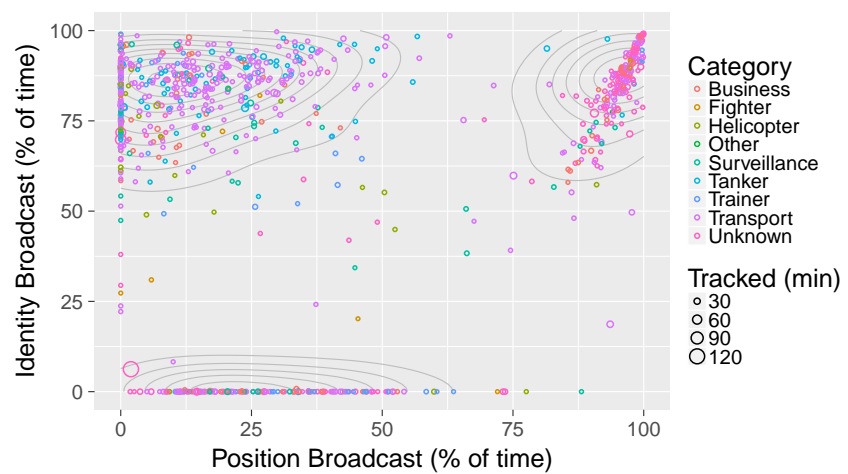

(a) Percentage of time where ADS-B position broadcast is enabled versus percentage of time where ID broadcast is enabled per country.

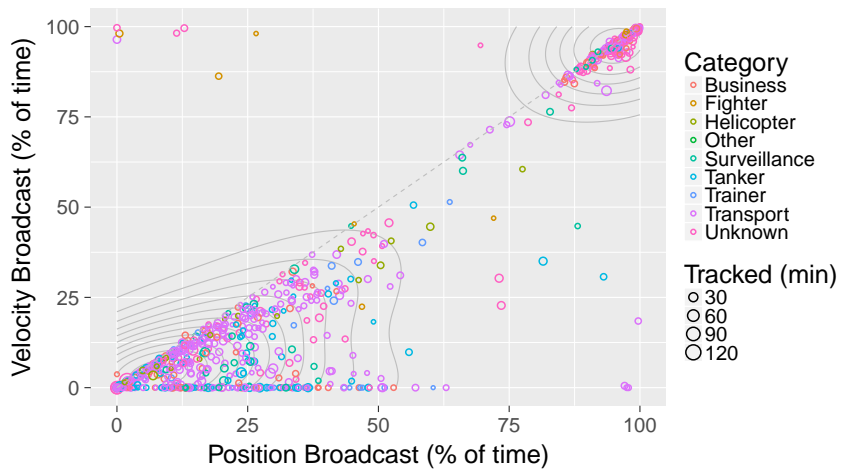

(b) Percentage of time where ADS-B position broadcast is enabled versus percentage of time where velocity broadcast is enabled per country.

Figure 8: Percentage of possible ADS-B broadcasts for position against identity and velocity. We also plot the category of aircraft and the amount of time said aircraft was tracked for.

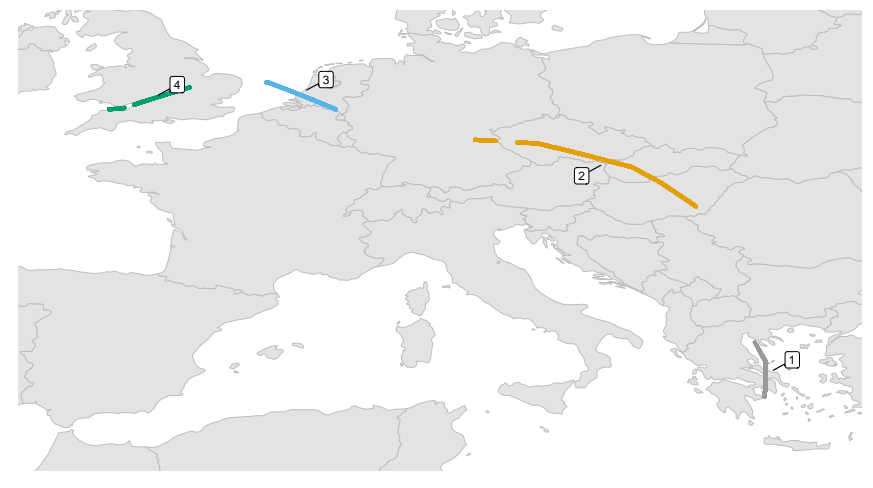

(a) Track of example military flight (via ADS-B)

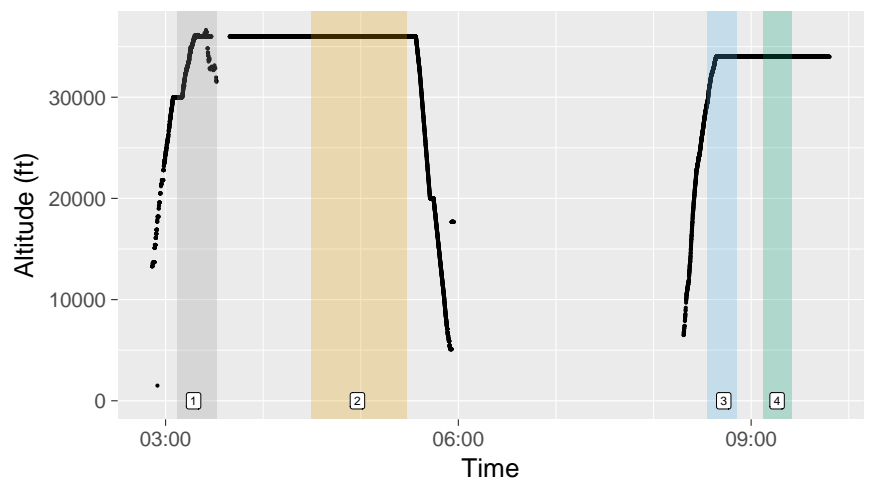

(b) Altitude profile of example military flight (via Mode S)

Figure 9: A military flight crossing Europe on the 20th of April, 2017. The flight started in Greece at around 1am UTC, had a 2:15h layover in Germany, and left OpenSky's coverage at 10am UTC in the UK. We assume that the aircraft was in OpenSky's reception area as long as there was Mode $S$ altitude data available.

value; other unfiltered crowd-sourced services exist, e.g. ADSB Exchange [19]. Indeed, they actively identify 'interesting' aircraft to the public, among them many military and other state aircraft.

Using OpenSky, we analyze the extent of tracking blocks active on such commercial websites for military-operated aircraft. Table III provides the breakdown of such blocks across the different military categories. Overall, almost $90 \%$ (5223 out of 6024) of all transponder-equipped aircraft tracked by us were not visible on commercial trackers, suggesting they use ASDI blocks or similar means.

All individual categories have a blocking rate of at least $80 \%$ (and up to $99.1 \%$ for the observed tanker aircraft) with the exception of UAV, which is not necessarily reliable because of the extremely small sample size.

Table IV illustrates the extent of blocking for the nonmilitary state aircraft in April 2017. In general, we can see that the overall ratio of blocked aircraft is below $40 \%$ and thus distinctively lower compared to military aircraft.

\begin{tabular}{|l|c|c|}
\hline Type & Not Blocked & Blocked \\
\hline Fighter & $9(2.2 \%)$ & $400(97.8 \%)$ \\
\hline Trainer & $195(14.4 \%)$ & $1161(85.6 \%)$ \\
\hline Tanker & $3(0.9 \%)$ & $322(99.1 \%)$ \\
\hline Transport & $339(20.0 \%)$ & $1352(80.0 \%)$ \\
\hline Business & $56(11.5 \%)$ & $430(88.5 \%)$ \\
\hline Surveillance & $17(7.9 \%)$ & $197(92.1 \%)$ \\
\hline UAV & $8(66.7 \%)$ & $4(33.3 \%)$ \\
\hline Helicopters & $146(15.8 \%)$ & $778(84.2 \%)$ \\
\hline Other/Uncategorized & $28(3.6 \%)$ & $579(96.4 \%)$ \\
\hline \hline All & $801(13.3 \%)$ & $5223(86.7 \%)$ \\
\hline
\end{tabular}

Table III: ADS-B equipage and state of visibility on commercial websites of military aircraft categories.

When comparing the different subtypes, aircraft used by the coast and border guard are more likely to be blocked because of their nature as surveillance aircraft. Police aircraft and in particular civil defense aircraft used for emergency and disaster response are less likely to not be displayed 


\begin{tabular}{|l|c|c|c|}
\hline Aircraft Type & Aircraft & Not Blocked & Blocked \\
\hline Police & 363 & $197(54.3 \%)$ & $166(45.7 \%)$ \\
\hline Coast Guard & 58 & $23(39.7 \%)$ & $35(60.3 \%)$ \\
\hline Border Guard & 22 & $8(36.4 \%)$ & $14(63.6 \%)$ \\
\hline Civil Defense & 204 & $166(81.4 \%)$ & $38(18.6 \%)$ \\
\hline Government & 404 & $234(57.9) \%$ & $170(42.1 \%)$ \\
\hline Other & 62 & $55(88.7 \%)$ & $7(11.3 \%)$ \\
\hline \hline All State A/C & 1113 & $683(61.4 \%)$ & $430(38.6 \%)$ \\
\hline
\end{tabular}

Table IV: Breakdown of state-related aircraft seen by OpenSky in April 2017 and their visibility on commercial tracking sites.

by commercial trackers. Interestingly, even 234 of the 404 government aircraft used by heads of state and high-ranking government officials were visible on commercial trackers at the time of writing. We can only speculate on the reasons for this presumed oversight, as they, too, typically have a great interest in movement privacy. Still, it may be the case that the awareness of the problem in the relevant departments is lower than in their respective countries' air forces and collaboration on such issues has not yet progressed far enough.

\section{B. Effectiveness of Privacy Measures}

The nature of state aircraft poses an interesting angle to consider Mode S and ADS-B deployment from. Our results in the previous sections indicate that there is a heightened location and movement privacy requirement for the military which is potentially undermined by the use of ADS-B. After many publications and news reports over recent years, ADS-B is now widely known to be cheap and easy to receive, thus making state aircraft potentially more vulnerable to comprehensive tracking.

This awareness may be the underlying reason why ADS$B$ is not mandated for many state and military aircraft to the same extent as for commercial aviation and why adoption rates among military actors are so low. As seen in Section $\mathrm{V}-\mathrm{B}$, there is a much more varied set of deployments of Mode S-based technologies, illustrated by a significant group of aircraft solely transmitting position with no identity broadcast—and equally, a large group regularly transmitting identity without position. Finally, as shown in Section VI, some military aircraft do not use it during take-off and approach phases in an attempt to conceal their destinations.

Alone, we argue that this reasoning has limited worth since with the same soft- and hardware equipment it is similarly easy to track Mode S, which the vast majority of state aircraft are mandated to use. While this would not allow the same granularity of tracking as with ADS-B, it is obviously trivial to estimate the destination airport from the altitude profiles broadcast using Mode S. For really ambitious actors with several sensors or a sensor network similar to OpenSky, it is furthermore possible to localize an aircraft sending out any signal by using multilateration or other time difference of arrival-based methods.

Similar thinking about privacy requirements seems to underlie the blocking of aircraft displays from commercial trackers discussed in this section. Like using Mode $\mathrm{S}$, it is ineffective in achieving the intended goal. In reality, all schemes to prevent the widespread tracking of sensitive aircraft using open ATC communication have become obsolete. Indeed, much relevant information can be obtained from leaky commercial trackers, social media feeds and dedicated websites such as ADS-B Exchange [19]. As military patterns can easily be detected through these sources, potentially sensitive strategic and operational information may be obtained. This leads to the conclusion that the impact of using insecure civil aviation protocols on military users is growing and new considerations have to be made if air forces want to regain their full movement privacy in the future.

\section{FUTURE PLANS}

The OpenSky Network is an ongoing effort that improves and extends its capabilities continuously. As of this writing, new sensors are being deployed around the world and existing sensors from the community become integrated. Apart from the ability to monitor, e.g., the progress of ADS-B deployment or Mode $\mathrm{S}$ usage, growth and increased receiver density will allow us to perform multilateration to additionally locate non-ADS-B aircraft. In addition, other sensors for wireless ATC technologies such as the surveillance protocols FLARM and UAT ADS-B or the general purpose datalinks ACARS and CPDLC are planned to be included in the OpenSky Network to provide a more complete picture of the global airspace. Besides integrating new technologies, we also aim at incorporating additional sources of metadata such as aircraft databases or weather data. Merging this metadata with the data provided by the sensors widens the range of possible applications and analyses in OpenSky considerably.

These goals, however, require the support of the community for sensor sponsoring and hosting and the willingness to share data. To join and support the OpenSky Network initiative, please refer to http://opensky-network.org.

\section{CONCLUSION}

In this paper, we analyzed the usage of Mode S and ADS-B by state and military aircraft in large areas in Europe, North America, and across all continents. The data used for this analysis is collected by the OpenSky Network, a research initiative which continuously monitors and records air traffic control communications using more than 700 Mode S sensors across the globe. We identified and categorized the state aircraft tracked by OpenSky by incorporating and comparing publicly available data sources. Based on our analysis, we can conclude that about $42.9 \%$ of all Mode S transponders seen in use by military aircraft have ADS-B capabilities, with considerable variation between countries. As this number is considerably lower than the current ADS-B deployment in civil aviation reported last year, there is certainly a long way to go to for the ongoing ADS-B deployment. 


\section{REFERENCES}

[1] International Civil Aviation Organization (ICAO), Convention on International Civil Aviation, 9th ed., 2006, Doc 7300/9.

[2] Civil/Military Cooperation in Air Traffic Management, International Civil Aviation Organization (ICAO), 2011, Cir 330.

[3] C. Spitzer, U. Ferrell, and T. Ferrell, Digital Avionics Handbook, 3rd ed. CRC Press, Sep. 2014.

[4] M. Schäfer, M. Strohmeier, M. Smith, M. Fuchs, R. Pinheiro, V. Lenders, and I. Martinovic, "OpenSky Report 2016: Facts and Figures on SSR Mode S and ADS-B Usage," in Proceedings of the 35th IEEE/AIAA Digital Avionics Systems Conference (DASC), Sept 2016.

[5] M. Strohmeier, M. Schäfer, M. Fuchs, V. Lenders, and I. Martinovic, "Opensky: A swiss army knife for air traffic security research," in Proceedings of the 34th IEEE/AIAA Digital Avionics Systems Conference (DASC), Sep. 2015.

[6] M. Schäfer, M. Strohmeier, V. Lenders, I. Martinovic, and M. Wilhelm, "Bringing Up OpenSky: A Large-scale ADS-B Sensor Network for Research," in Proceedings of the 13th IEEE/ACM International Symposium on Information Processing in Sensor Networks (IPSN), Apr. 2014.

[7] M. Strohmeier, M. Smith, M. Schäfer, V. Lenders, and I. Martinovic, "Crowdsourcing security for wireless air traffic communications," in Proceeding of the 9th International Conference on Cyber Conflict (CyCon), Jun. 2017.

[8] "PlanePlotter," accessed on 2017-07-08. [Online]. Available: http: //www.coaa.co.uk/planeplotter.htm

[9] J. Sun, "World Aircraft Database," accessed on 2017-07-08. [Online]. Available: http://junzisun.com/adb/

[10] R. D. Kloth, "Airframes.org," accessed on 2017-07-08. [Online]. Available: http://www.airframes.org/

[11] Federal Aviation Administration, "Aircraft Registry - Releasable Aircraft Database Download," 2017, accessed on 2017-02-19. [Online]. Available: https://goo.gl/wpCxJt

[12] D. Cenciotti, "U.S. Air Force RQ-4 Global Hawk drone flew over Ukraine with transponder turned on for everyone to see," The Aviationist, Oct. 2016, accessed on 2017-04-19. [Online]. Available: https://goo.gl/nfDOFU

[13] European Organisation For the Safety of Air Navigation (EUROCONTROL), Mode $S$ - Harmonisation of the Transition Arrangements for State Aircraft - Process and Procedures for the Managing of the Mode S Compliance Status and the Exemptions Granted to State Aircraft, 1st ed., Jan. 2005.

[14] J. Scardina, "Overview of the FAA ADS-B Link Decision," Federal Aviation Administration, Jun. 2002.

[15] M. Strohmeier, M. Smith, M. Schäfer, V. Lenders, and I. Martinovic, "Assessing the impact of aviation security on cyber power," in Proceedings of the 8th International Conference on Cyber Conflict (CyCon), 2016.

[16] M. Smith, D. Moser, M. Strohmeier, V. Lenders, and I. Martinovic, "Analyzing privacy breaches in the aircraft communications addressing and reporting system (acars)," arXiv preprint arXiv:1705.07065, 2017.

[17] National Business Aviation Administration, "Blocking display of Aircraft Situation Display to Industry (ASDI) data," 2016. [Online]. Available: https://www.nbaa.org/ops/security/asdi/

[18] T. Webster, "Is a war on live flight tracking coming?" Mar. 2017, accessed on 2017-04-19. [Online]. Available: https://tonywebster.com/ 2017/03/war-on-adsb-receivers/

[19] D. Streufert, "ADS-B Exchange," 2016, accessed on 2017-04-19. [Online]. Available: https://www.adsbexchange.com/ 